\title{
2D Hexagonal Boron Nitride (2D-hBN) Explored for the Electrochemical Sensing of Dopamine
}

\author{
Aamar F. Khan ${ }^{1}$, Dale A. C. Brownson ${ }^{1}$, Edward P. Randviir ${ }^{1}$, \\ Graham C. Smith ${ }^{2}$, and Craig E. Banks ${ }^{1 *}$
}

${ }^{1}$ Faculty of Science and Engineering, Manchester Metropolitan University, Chester Street, Manchester M1 5GD, U.K.

* Author for correspondence

${ }^{2}$ Faculty of Science and Engineering, Department of Natural Sciences, University of Chester, Thornton Science Park, Pool Lane, Ince,

Chester CH2 4NU, U.K.

\begin{abstract}
:
Crystalline 2D hexagonal boron nitride (2D-hBN) nanosheets are explored as a potential electrocatalyst toward the electroanalytical sensing of dopamine (DA). The 2D-hBN nanosheets are electrically wired via a drop-casting modi fication process onto a range of commercially available carbon supporting electrodes, including glassy carbon (GC), boron-doped diamond (BDD), and screen-printed graphitic electrodes (SPEs). 2D-hBN has not previously been explored toward the electrochemical detection/electrochemical sensing of DA. We critically evaluate the potential electrocatalytic performance of 2D$\mathrm{hBN}$ modified electrodes, the effect of supporting carbon electrode platforms, and the effect of "mass coverage" (which is commonly neglected in the 2D material literature) toward the detection of DA. The response of 2D-hBN modified electrodes is found to be largely dependent upon the interaction between $2 \mathrm{D}-\mathrm{hBN}$ and the underlying supporting electrode material. For example, in the case of SPEs, modification with 2D-hBN (324 ng) improves the electrochemical response, decreasing the electrochemical oxidation potential of DA by $\sim 90 \mathrm{mV}$ compared to an unmodified SPE. Conversely, modification of a GC electrode with 2D-hBN (324 ng) resulted in an increased oxidation potential of DA by $\sim 80 \mathrm{mV}$ when compared to the unmodified electrode. We explore the underlying mechanisms of the aforementioned examples and infer that electrode surface interactions and roughness factors are critical considerations. $2 \mathrm{D}-\mathrm{hBN}$ is utilized toward the sensing of DA in the presence of the common interferents ascorbic acid (AA) and uric acid (UA). 2D-hBN is found to be an effective electrocatalyst in the simultaneous detection of DA and UA at both pH 5.0 and 7.4. The peak separations/resolution between DA and UA increases by $\sim 70$ and $50 \mathrm{mV}$ (at pH 5.0 and 7.4, respectively, when utilizing $108 \mathrm{ng}$ of 2D-hBN) compared to unmodified SPEs, with a particularly favorable response evident in $\mathrm{pH}$ 5.0, giving rise to a significant increase in the peak current of DA. The limit of detection $(3 \sigma)$ is found to correspond to $0.65 \mu \mathrm{M}$ for DA in the presence of UA. However, it is not possible to deconvolute the simultaneous detection of DA and AA. The observed electrocatalytic effect at $2 \mathrm{D}-\mathrm{hBN}$ has not previously been reported in the
\end{abstract}


literature when supported upon carbon or any other electrode. We provide valuable insights into the modifier - substrate interactions of this material, essential for those designing, fabricating, and consequently performing electrochemical experiments utilizing 2D-hBN and related 2D materials.

$\mathrm{D}$ opamine (DA) is a catecholamine that plays a vital role in the functioning of numerous biological systems. ${ }^{1,2}$ Studies suggest abnormal levels of DA can lead to neurological disorders such as Parkinson's disease and schizophrenia. ${ }^{1,3}$ There have been numerous efforts directed toward the electrochemical detection of DA. ${ }^{4-6}$ However, DA tends to coexist alongside ascorbic acid (AA), which leads to overlapping voltammetric signals, giving rise to limitations when detecting these compounds. ${ }^{7}$ As a result, current research is focused on improving the analytical sensitivity and selectivity for these two important compounds. ${ }^{8,9}$

Research using gold $(\mathrm{Au})$, platinum $(\mathrm{Pt})$, and glassy carbon (GC) electrodes does not allow the sufficient differentiation between the anodic oxidation peak potentials of DA and $\mathrm{AA}^{10}$ Furthermore, because of the oxidized species of DA and AA residing on the electrode surface, these electrodes are prone to fouling, leading to reduced sensitivity, inhibited linear ranges, and inadequate electroanalytical capabilities. ${ }^{10}$ As such, an electrode material that improves the electrochemical detection capabilities of DA is highly sought, particularly while resisting both chemical and electrochemical surface fouling ${ }^{11}$ and in the case of applied sensors, where AA and uric acid (UA) are commonly found to coexist within sample matrixes. ${ }^{12}$

In previous research, Deng et al. ${ }^{10}$ showed that AA and DA activation potentials could be distinguished through manipulation of GC electrodes using boron-doped carbon nanotubes (BCNTs), resulting in an anodic peak separation for AA and DA of $\sim 238 \mathrm{mV}$. This was highly beneficial in comparison to a "regular" carbon nanotube (CNT) modified GC electrode that exhibited a peak separation of $\sim 122 \mathrm{mV} .^{10}$ This enhanced 
Table 1. Comparison of Various Electrode Materials Utilized Towards the Detection of DA in the Presence of UA and AA

\begin{tabular}{|c|c|c|c|c|c|}
\hline electrode material & $\begin{array}{l}\text { electrocatalytic } \\
\text { (with regands to) }\end{array}$ & $\begin{array}{l}\text { dopamine Lon } \\
(\mu \mathrm{M})\end{array}$ & $\begin{array}{l}\text { explored in the prosence } \\
\text { of }\end{array}$ & $\begin{array}{l}\text { dectrochemizal } \\
\text { method }\end{array}$ & ref \\
\hline nitrogen doped graphene & GC & 0.25 & UA, AA & DPV & 16 \\
\hline ditosan-graphene & GC & 1.00 & $\mathrm{UA}, \mathrm{AA}$ & DPV & 47 \\
\hline carbon ivenic liquid clectrode & CPE & 1.00 & UA, Mn & DPV & 48 \\
\hline AuNPspMos, & GC & 0.05 & UA, AA & DPV & 18 \\
\hline Pt-Am hytrid & GC & 240 & UA, AA & DPV & 49 \\
\hline Poly(Evans blue) & cc & 0.25 & Un, Mn & DPV & so \\
\hline AuNPs- $\beta \mathrm{CD}$-graphene & GC & a.15 & UA, AA & swv & S1 \\
\hline $\begin{array}{l}\text { ondered mesoporous carboa/Nafiven composite } \\
\text { film }\end{array}$ & GC & 0.50 & UA, A A & DPV & 52 \\
\hline functionalined graphene & bare graphite & 0.25 & UA, AA & DPV & 53 \\
\hline 2DO-hBN & SPE. & 0.65 & $\mathrm{UN}, \mathrm{M}^{6}$ & DPV & this work \\
\hline
\end{tabular}

${ }$ Key: AuNP@MoS ${ }_{2}$ gold nanoparticle modified molybdenum disulfide; AuNPs- $\beta$ CD-Giraphene, gold nanopartides- $\beta$ cydodextrin-graphene; 2D hBN, 2D hexagonal boron nitride; GC, glassy carbon electrode; CPE, carbon paste electrode; functionalized graphene synthesized by solvothermal reduction of colloidal dispersions of graphite oxide; UA, uric acid; $\mathrm{A \Lambda}$, ascorbic acid; DPV, differential pulse voltammetry; SWV, square wave voltammetry. ${ }^{\mathrm{DA}}$ and $\mathrm{AA}$ signal deconvolution is not possible upon simultaneous detection.

response with BCNTs was attributed to the edge plane sites of the CNTs and a change in electronic structure, with borondoping improving the electron transfer kinetics of the CNTs. $10,13,14$ Similarly, Medeiros et al. ${ }^{7}$ conducted studies toward the simultaneous detection of DA and AA utilizing carbon nitride electrodes $\left(\mathrm{CN}_{x}\right)$ and observed an anodic peak separation of $\sim 330 \mathrm{mV}$, attributed to the anodic pretreatment (APT) of the $\mathrm{CN}_{x}$ in an alkaline medium. ${ }^{7}$ Furthermore, Wang et al. ${ }^{15}$ reported highly sensitive methods for the detection of DA, using Nafion/carbon nanotube coated poly(3-nethylthiophene) modified electrodes. ${ }^{15}$ Such methods require extensive sample pretreatment and use complex electrode composites but are promising nevertheless. An overview of the different materials utilized in the simultaneous detection of DA, AA, and UA can be found in Table 1.

Numerous newly emerged 2D nanomaterials have been considered for the detection of DA in recent years, such as graphene, ${ }^{6,16,17}$ molybdenum disulfide $\left(\mathrm{MoS}_{2}\right)^{18}$ and $\mathrm{MoS}_{2}$ decorated with gold nanoparticles. ${ }^{18}$ However, to our knowledge, a lesser researched nanomaterial is crystalline hexagonal boron nitride (2D-hBN). Crystalline $2 \mathrm{D}-\mathrm{hBN}$ is a $2 \mathrm{D}$ material comprising equal quantities of boron and nitrogen atoms arranged in a hexagonal structure; similar to graphene, hence it is referred to as "white graphene" ${ }^{19}$ 2D-hBN contains strong $\sigma$ bonds and weak van der Waals forces, ${ }^{20}$ resulting in a nanomaterial that displays high thermal conductivity and is mechanically strong and yet lubricating. ${ }^{21} 2 \mathrm{D}-\mathrm{hBN}$ has been utilized as an electrocatalyst in applications such as the oxygen reduction reaction (ORR $)^{22}$ and in solar energy conversion by photoredox catalysis. ${ }^{23}$ This reported electrocatalytic performance might prove beneficial for separating the voltammetric signals of DA, $\mathrm{M}$, and $\mathrm{UA}$; such an application has not previously been considered for this novel $2 \mathrm{D}$ material.

In this paper, we explore for the first time the utilization of 2D-hBN as a potential electrocatalyst toward the detection of DA when immobilized upon a range of commercially available graphitic electrodes/substrates while producing multiple massrelated coverage studies (commonly overlooked in the literature). This work then considers 2D-hBN modified graphitic substrates toward the improved simultaneous detection of DA, AA, and UA. 2D-hBN has not previously been reported as a beneficial electrocatalytic material when immobilized upon carbon or any other platforms applied toward the electrochemical detection of DA (and in the presence of AA and UA). This research provides new insights into the exploration of 2D-hBN's electrochemical properties, performance, and applicability.

\section{EXPERIMENTAL SECTION}

All chemicals were of analytical grade and were used as received from Sigma-Aldrich without any further purification. All solutions were prepared with deionized water of resistivity not less than $18.2 \mathrm{M} \Omega \mathrm{cm}$ and were vigorously degassed prior to electrochemical measurements with high purity, oxygen free nitrogen.

Voltammetric measurements were performed using an "Autolab PGSTAT 101" (Metrohm Autolab, The Netherlands) potentiostat. All measurements were conducted using a conventional three electrode system. The working electrodes were a $3 \mathrm{~mm}$ diameter screen-printed graphite electrode (SPE), a glassy carbon (GC) electrode (3 mm diameter, BAS), and a boron-doped diamond (BDD) electrode ( $3 \mathrm{~mm}$ diameter, BAS). A platinum wire and a saturated calomel electrode (SCE) were used as counter and reference electrodes, respectively. 2DhBN modified electrodes were prepared by drop-casting aliquots of an ethanol solution containing pristine flakes of 2D-hBN onto the required working electrode with a micropipette. After $30 \mathrm{~min}$, the ethanol evaporated (at ambient temperature) and the modified electrodes were ready for use (or for further modification). Prior to modification or commencing experiments, the GC, BDD working electrodes (and SPEs where necessary) were polished with "Kemet diamond spray" (1 and 1/4 $\mu \mathrm{m}$ sized particles) and washed extensively with deionized water to remove any remaining surface contaminants.

The SPEs consist of a graphite working electrode and were fabricated in-house. Fabrication details and appropriate characterization can be found in the Supporting Information, along with the reported electrochemical kinetic properties of unmodified GC and BDD electrodes to allow future researchers to benchmark the system utilized.

The 2D-hBN utilized was commercially procured from "Graphene Supermarket" (Reading, MA) and is known as "Boron Nitride Pristine Flakes" comprised entirely of pristine 2D-hBN nanoscale crystals dispersed in ethanol $\left(5.4 \mathrm{mg} \mathrm{L}^{-1}\right)$ that have not been oxidired, reduced, or chemically modified in anyway and are free from surfactants, ${ }^{24,25}$ The exact details of the fabrication process are proprictary information. The $2 \mathrm{D}$ - 


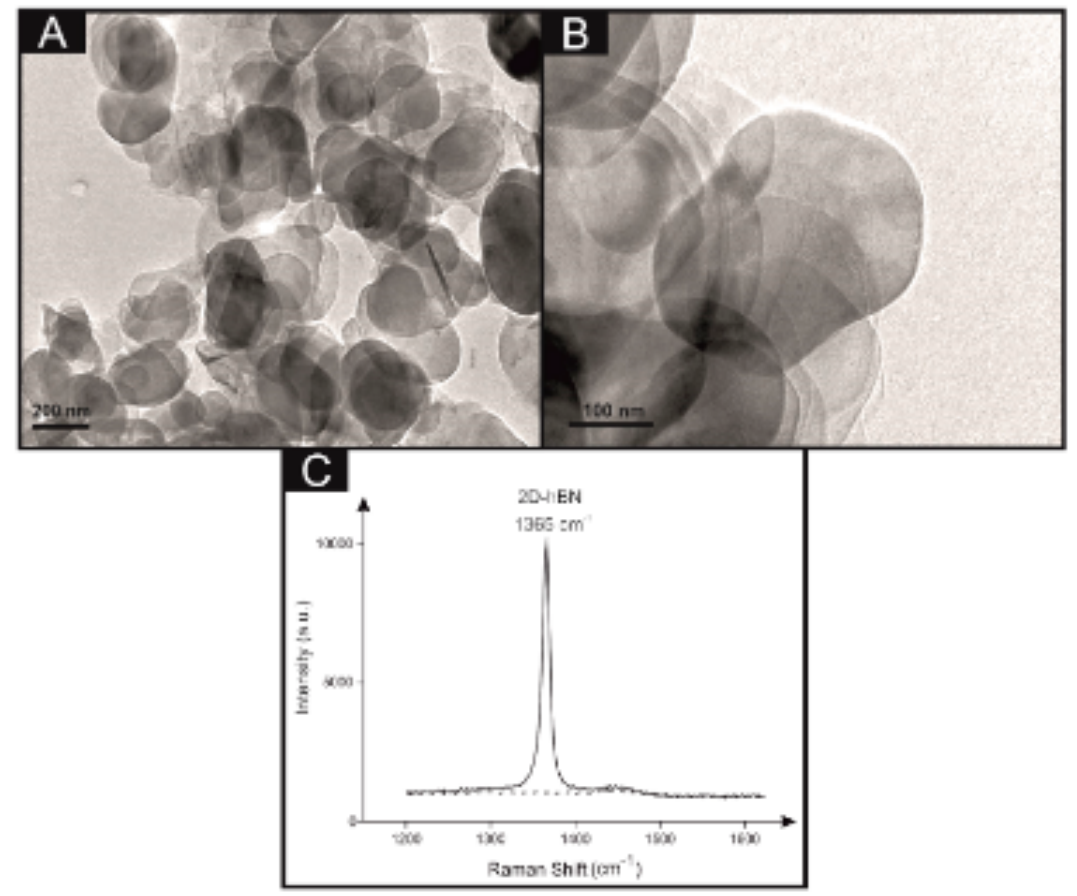

Figure 1. Typical TEM images of 2D-bBN nanosheets. Scale bars are $200 \mathrm{~nm}(\mathrm{~A})$ and $100 \mathrm{am}$ (B). (C) A typical Raman spectra obtained for 2DhIN (solid line) immobilized upon a supporting silicon wafer is presented, where the dotted line depicts the Raman spectra of the silicon substrate upon which $2 \mathrm{D}$ hBN is deposited.

hBN platelets are reported to have an average particle size (lateral) of $\sim 50-200 \mathrm{~nm}$, a thickness of between 1-S monolayers in solution, and a purity (in the dry phase) of $>99 \%$.

Physicochemical characterization of the 2D-hBN was performed utilizing Raman spectroscopy, X-ray diffraction (XRD), transmission electron microscopy (TFM), scanning electron microscopy (SEM), energy-dispersive X-ray (EDX) analysis, X-ray photoclectron spectroscopy (XPS), and white light profilometry. Details of the equipment utilized are reported in the Supporting Information.

Physicochemical Characterization of 2D-hBN. TEM, SEM, EDX, XPS, XRD, and Raman spectroscopy were conducted to ascertain the quality/properties of our commerdally procured 2D-hBN. TEM images are depicted in Figure 1 and indicate that the $2 \mathrm{D}-\mathrm{hBN}$ platelets have an average partide size (lateral) of $\sim 200 \mathrm{~nm}$, which agrees with the commercial manufacturer. ${ }^{24}$ Figgure 1C depicts a Raman spectra of $2 \mathrm{D}-\mathrm{hBN}$ immobilized upon a silicon wafer, performed in order to verify the structural characterization. A characteristic Raman peak at $1365 \mathrm{~cm}^{-1}$ is evident, due to the $\mathrm{E}_{25}$ phonon mode, ${ }^{26}$ which is in excellent agreement with previous literature. ${ }^{26}$ Peak analysis indicates that $2 \mathrm{D}-\mathrm{hBN}$ comprises of between 2-4 layers when immobilized upon a surface (a silicon wafer in this case). Because of overlapping signals occurring between $2 \mathrm{D}-\mathrm{hBN}$ and carbon, Raman spectra is not easily deconvoluted/observed for 2D-hBN when one material is deposited upon the other. Thus, it is not feasible to refer to 2D-hBN modified graphite electrodes in terms of the number of layers immobilized upon the electrode surface in question, but rather the mass of $2 \mathrm{D}$. hBN immobilized. Figure S-1B,C show Raman map compar- isons of an unmodified and a $324 \mathrm{ng}$ 2D-hBN modified SPE. Figure S-1B shows a smooth surface with uniform and low intensity Raman signal. Conversely, Figure S-1C depicts a general darker color, indicating monolayer 2D-hBN deposition, with numerous areas appearing as black dots (that are suggestive of higher/thicker layers of $2 \mathrm{D}$-hBN immobilization). Note that Raman intensities were recorded at the wavenumber (1365 $\mathrm{cm}^{-1}$ ) corresponding to the peak observed in Figure 1C. Thus, 2D-hBN modified electrodes significantly differ from that of the underlying electrode substrate, and it is clear that the method chosen (drop-casting) to modify and "electrically wire" to the $2 \mathrm{D}-\mathrm{hBN}$ is sufficient for its electrochemical exploration.

XPS analysis was performed on the $2 \mathrm{D}-\mathrm{hBN}$ and as depicted in Figure S-2, the XPS spectra showed the presence of a single component at $190.8 \mathrm{eV}$ in the $\mathrm{B} 1 \mathrm{~s}$ spectrum, with the $\mathrm{N} 1 \mathrm{~s}$ spectrum exhibiting a main peak at $398.4 \mathrm{eV}$. Observations of both peaks are in agreement with previous literature. 27 The stoichiometry of 1:1 for $\mathrm{B} / \mathrm{N}$ is noted, and the binding energies for the $\mathrm{B}$ 1s and $\mathrm{N}$ is photoclectron peaks agree well with the expected values for 2D-hBN. Table S-1 exhibits the full surface composition of the dried ethanol suspension of $2 \mathrm{D}-\mathrm{hBN}$ on $\mathrm{Si}(111)$ from the XPS analysis. Furthermore, detailed analysis of the spectra and other contributions from the support surface are presented in the Supporting Information.

It has been reported that metallic impurities in CNTs can contribute to the electrocatalysis observed at such modifiedelectrodes ${ }^{25}$ It is important to consider the possible presence of metallic impurities for the case of 2D-hBN which, if present, may contribute to the observed voltammetry. FDX analysis was performed on a single platelet of 2D-hBN (Figure S-3) and indicated a composition of $11.40 \%$ atomic boron and $9.93 \%$ 


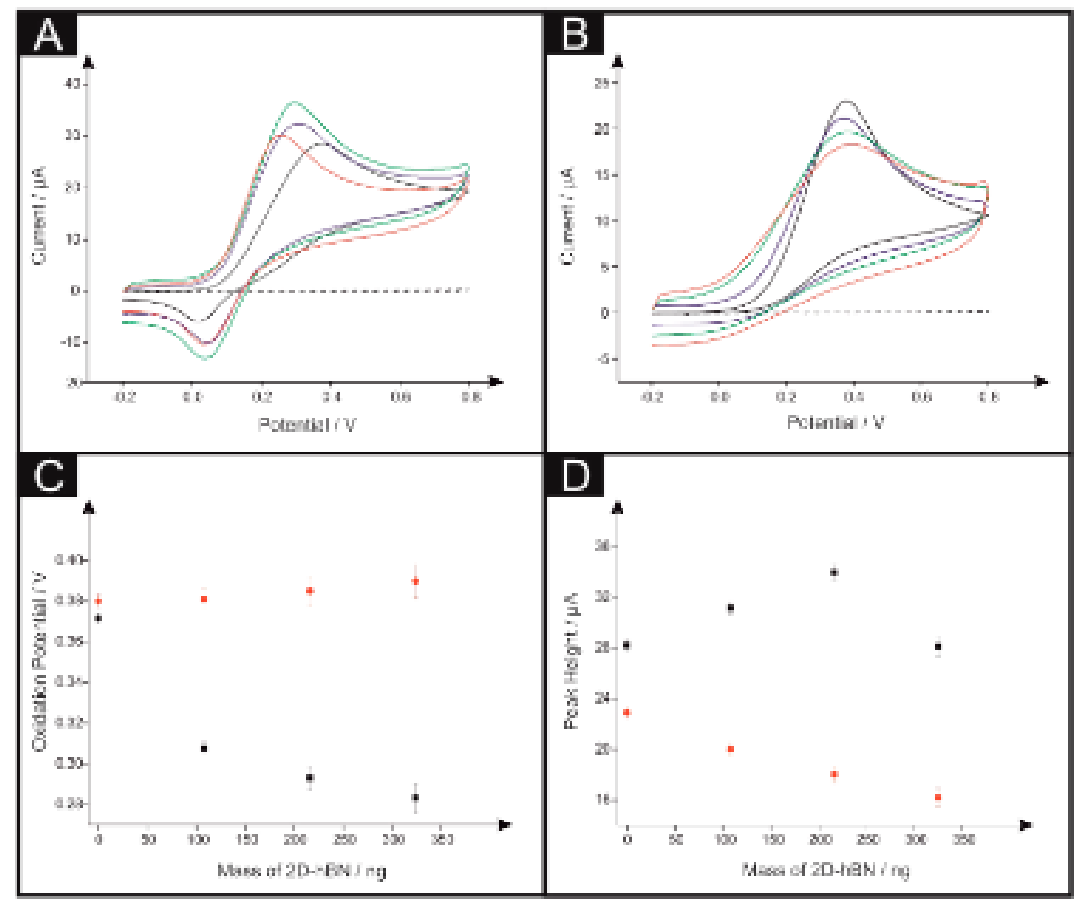

Figure 2. Typical cydic voltammograms recorded in (A) $1 \mathrm{mM}$ DA and (B) $1 \mathrm{mM}$ AA, pII 7.4 PBS with unmodified SPEs (black) and 2D-hBN modified electrodes. Masses of modification as follows: 108 (blue), 216 (green), and $324 \mathrm{ng}$ (red) immobilized. The dashed line represents the PBS blanks, respectively. Analysis of the cyclic voltammograms is presented in the form of plot (C) illustrating oxidation potential of DA (black square) and AA (red circle) vs mass of 2D-hBN immobilized and plot (D) depicting peak current of DA (black square) and AA (red circle) vs mass of 2D. hBN immobilixed. Scan rate: $100 \mathrm{mV} \mathrm{s}^{-1}$ (vs SCE). Each data point (C and D) is the average and standard deviation $(N=3)$.

atomic nitrogen, corresponding to the stoichiometry between $\mathrm{B} / \mathrm{N}$ of $\sim 1: 1$. Further scrutiny of the EDX data reveals a $78.67 \%$ contribution/component of atomic silicon (Si), which originates from the substrate upon which $2 \mathrm{D}-\mathrm{hBN}$ is immobilized. Analysis confirms the absence of impurities within our commercially obtained 2D-hBN. Hence, the electrochemical response is not dominated by such metallic constituents but rather is due solely to that of the 2D-hBN present.

Finally, an XRD spectrum is presented in Figure S-4 and reveals the 2D-hBN crystals are oriented in the (002) direction, with a characteristic peak occurring at $26.7^{\circ} .{ }^{29}$

The combined surface and physicochemical analysis presented above indicates the presence high quality and high purity $2 \mathrm{D}-\mathrm{hBN}$ nanosheets. Interested readers should note that we have previously reported the electrochemical characterization (in terms of the heterogeneous electron transfer properties) of the 2D-hBN utilized herein toward a range of redox systems (see ref S4). We now explore this material's electrocatalytic properties/potential toward the electrochemical oxidation/detection of DA.

\section{RESULTS AND DISCUSSION}

Dopamine Electrochemistry. The electron transfer reactions between $2 \mathrm{D}-\mathrm{hBN}$ and $\mathrm{D} \Lambda$ were first studied using unmodified SPE, GC, and BDD electrodes in order to benchmark our system. Figure S-S depicts the respective cyclic voltammograms obtained toward the oxidation of $1 \mathrm{mM}$ DA (in pH 7.4 phosphate buffer solution (PBS)). The anodic oxidation potential utilizing SPEs is obtained at $+0.37 \mathrm{~V}$ corresponding to the electrochemical oxidation of DA, which agrees well with previous literature. ${ }^{30}$ Comparatively, the GC electrode exhibits an observed peak at $+0.31 \mathrm{~V}$ and the BDD electrode at $+0.45 \mathrm{~V}$, with both in agreement with previous studies. ${ }^{31,32}$ These findings indicate that the GC electrode exhibits the lowest (most beneficial) potential required for the electrochemical oxidation of DA. However, the SPEs exhibits a greater peak current, offering a more favorable peak for electroanalytical application and thus potentially higher sensitivity at lower concentrations. The BDD electrode exhibits the highest potential and lowest peak current toward the detection of $\mathrm{DA}$, as one would expect due to the chemical nature and reactivity of the BDD electrode. 14,33,34

The electrochemical oxidation of DA was next considered using electrodes modified with increasing $2 \mathrm{D}-\mathrm{hBN}$ masses (a coverage study), ranging from 108 to $324 \mathrm{ng}$. Figure $2 \mathrm{~A}$ depicts typical cyclic voltammograms toward DA detection using 2DhBN modified SPFs, where it is evident that increasing $2 \mathrm{D}-\mathrm{hBN}$ additions upon the SPE's surface beneficially lowers the potential required to electrochemically oxidize DA (a catalytic response). The observed peak currents also increase; however, this occurs until a mass of $324 \mathrm{ng}$ of $2 \mathrm{D} \cdot \mathrm{hBN}$ is reached and thus is mass/coverage dependent. Analysis of these voltammograms is presented in Figure 2C, where overall, a decrease in oxidation potential required for DA is observed of up to $90 \mathrm{mV}$ (compared to the bare electrode) upon modification of an SPE with 324 ng of $2 \mathrm{D}-\mathrm{hBN}$, suggesting that $2 \mathrm{D}-\mathrm{hBN}$ exhibits electrocatalytic activity toward DA. Previous studies using 2D nanomaterials (such as graphene modified EPPG and BPPG 
electrodes) toward $\mathrm{D} \Lambda$ detection have shown the activation potential increases, indicating not such a beneficial response, contrary to what we observe herein. $25,35,36$

Figure 2D depicts the eflect of 2D-hBN deposition upon a SPE when considering the peak current/height observed for DA. An increase in the peak current is observed in contrast to the unmodified SPI, with an overall increase of from 28.2 to $34.0 \mu \mathrm{A}$ evident when immobilizing $216 \mathrm{ng}$ of $2 \mathrm{D}-\mathrm{hBN}$. However, upon the highest deposition of $324 \mathrm{ng}$ of $2 \mathrm{D}-\mathrm{hBN}$, a decreased peak current is observed (to $28.1 \mu \Lambda$ ); one reported explanation for this response is that a "critical mass" of modification is achieved and surpassed whereby the 2D-hBN nanosheets can no longer support themselves upon the electrode surface and delaminate/detach when utilized. . $^{37,35}$ However, given that the potential continues to decrease (thus a continued electrocatalytic response), this may not be the case herein and we consider the response in more detail later.

Next, we consider the electrochemical implications of modifying $2 \mathrm{D}-\mathrm{hBN}$ onto smooth underlying electrode surfaces. In contrast to $2 \mathrm{D}-\mathrm{hBN}$ modified SPEs, the effect of $2 \mathrm{D}-\mathrm{hBN}$ deposition upon GC and BDD electrodes toward the oxidation of DA was found to differ significantly. Full interpretation of the electrochemistry is presented in the Supporting Information; however, it is clear that immobilization of $2 \mathrm{D}-\mathrm{hBN}$ upon such smooth supporting surfaces inhibits the observed electrocatalytic effects that were prominent above. In both cases, 2.D. hBN increases the oxidation potential required for the detection of DA and lowers the observed peak currents. We infer that the observed "potential" electrocatalytic effect of 2DhBN is dependent on the underlying support surface and more specifically surface roughness. Evidently, 2D-hBN modified SPEs (with rough surface morphologies) exhibit an electrocatalytic effect toward DA oxidation in comparison to unmodified SPEs. In contrast, modifications of $2 \mathrm{D}$-hBN upon smooth electrodes (GC and BDD) exhibit an increased (detrimental) potential for DA oxidation and decreased peak currents. This indicates that a smoother supporting electrode surface does not exhibit the same favorable interaction with 2DhBN as noted above (for the SPE), resulting in poor electrochemical performance. This likely originates as a result of $2 \mathrm{D}-\mathrm{hBN}$ detaching from the polished GC/BDD electrode surface, given the unfavorable interaction, reducing the sensitivity of DA detection. Whatever the cause of this observation, the phenomena is highly fascinating and will likely require further in-depth study that is outside the scope of this paper.

To offer preliminary insight into the above phenomena, SEM images were obtained to assess how the $2 \mathrm{D}-\mathrm{hBN}$ platelets prefer to reside upon smooth and rough SPE surfaces. Figure S$\mathrm{BA}, \mathrm{B}$ depicts the surface morphology of unpolished and polished SPEs. It is evident that the polishing process produces a smoother electrode surface via reducing the number of contours and ridges present, ${ }^{39}$ with a decrease in the quantity of the small binder particles covering the surface (used in the fabrication procedure to adhere the graphite-ink/paste together). Figure S-8C-F shows how changes in the SPE's morphology alter how $2 \mathrm{D}$-hBN rests upon the surface. In Figure S-8C, the 2D-hBN platelets are visible and appear as discs that collect preferentially around contours and ridges on the rough SPE surface. Conversely, Figure S-8D shows the platelets on a smooth surface collect in similar areas but also indicates large areas of underlying substrate where the platelets have not rested (in this case the surface is apparently not favorable to 2D-hBN interaction). With Figure S-8E,F highlighting this phenomenon further, it appears that 2DhBN prefers to reside upon a rougher electrode surface.

To further validate our inferences regarding the effectiveness of 2D-hBN interaction on rough and smooth electrode surfaces, the roughness factor $\left(R_{\mathrm{F}}\right)$ values for unmodified and 2D-hBN modified SPlis and GC electrodes were calculated using a double layer capacitance technique (full details and analysis can be found in the Supporting Information). ${ }^{37,40}$ Figures S-9 and S-10 depict the analysis of unmodified, 108, 216 , and $324 \mathrm{ng} 2 \mathrm{D}-\mathrm{hBN}$ modified SPEs and the $R_{\mathrm{F}}$ values obtained are 1.0, 31.6, 39.4, and 49.9, respectively. It is dear that the roughness factor significantly increases following 2DhBN modification of SPEs. Thus, it is revealed that 2D-hBN modification of SPEs exhibits excellent adherence upon the electrode surface, likely due to SPIs providing a high level of surface roughness, which may prove to be beneficial.

Comparatively, the $R_{F}$ values obtained for unmodified and 2D-hBN modified GC electrodes significantly differ. Figures S11 and S-12 present the analysis of unmodified, 108, 216, and $324 \mathrm{ng} 2 \mathrm{D}$-hBN modified GC electrodes, revealing the $\boldsymbol{R}_{\mathrm{F}}$ values of $1.00,1.36,2.00$, and 1.12 , respectively. It is dear that 2D-hBN adherence with a GC electrode is significantly lower when contrasted to an SPE. This adds further weight toward our inference that a smooth surface, such as a GC electrode, would be less likely to form strong interactions with $2 \mathrm{D}-\mathrm{hBN}$; whereas the rough/ridged surface of a SPE may increase the substrate interaction and thus appears to be beneficial.

White light profilometry was utilized to compare the surface topography of GC and both polished/unpolished SPE substrates. The surface of an unpolished SPE exhibited a root mean squared value of the heights over the whole surface (SQ) of $1338.8 \mathrm{~nm}$, which was significantly rougher than that of the GC $(7.6 \mathrm{~nm})$ and polished SPE $(806.6 \mathrm{~nm})$ alternatives, see Figure S-13. Surface topography was also explored following modification with $108 \mathrm{ng}$ of $2 \mathrm{D}$-hBN upon the said electrodes (Figure S-13). Immobilization of $2 \mathrm{D}-\mathrm{hBN}$ upon the GC electrode resulted in a rougher surface, with an increased SQ value of $35.1 \mathrm{~nm}$. This was also the case when utilizing the polished SPE, with an increased SQ value of $842.4 \mathrm{~nm}$ compared to the unmodified surface. Interestingly, 2D-hBN immobilization upon an unpolished SPE resulted in a significantly larger SQ value increase, from $1338.8 \mathrm{~nm}$ (unmodified) to $1752.9 \mathrm{~nm}$ (108 $\mathrm{ng}$ of $2 \mathrm{D}-\mathrm{hBN}$ ). Clearly, although a minimal increase in roughness is observed with the addition of 2D-hBN onto GC and polished SPEs (smooth surfaces), immobilization upon an unpolished SPE (an initially rough surface) gives rise to a substantial increase in surface roughness. This observation confirms insight gained through the $R_{\mathrm{F}}$ values and SFIMs analyned above and strengthens the inference that $2 \mathrm{D}-\mathrm{hBN}$ adheres much more readily to a rougher underlying surface (contrary to a smooth surface).

The above observations suggests that, in contrast to GC and BDD alternatives, modification of 2D-hBN onto SPlis gives rise to the most synergistic and resultantly beneficial electrocatalytic response toward the oxidation of DA (with an increased peak current and a reduction of up to $90 \mathrm{mV}$ in the peak potential). Given this result, we now focus upon exploring the dear electrocatalytic effect exhibited when immobilizing 2D-hBN onto a SPE as an underlying supporting surface and aim to obtain a favorable analytical response.

Ascorbic Acid and Dopamine Electrochemistry. The electrochemical detection of $1 \mathrm{mM}$ ascorbic acid (AA) in pH 
7.4 PBS was explored utilizing cyclic voltammetry at $2 \mathrm{D}-\mathrm{hBN}$ modified SPEs. Inspection of Figure $2 \mathrm{~B}$ reveals that an unmodified SPE activates the oxidation potential of AA at $+0.38 \mathrm{~V}$, with a peak current of $22.9 \mu \mathrm{A}$. Upon modification of the SPE with 2D-hBN (range, $108-324$ ng; see Figure 2B), it is dear that there is no significant alteration/change in the resulting oxidation potential of AA; however, a notable decrease in the peak current is evident (see Figure $2 \mathrm{C}, \mathrm{D}$ ). Thus, unlike the response obtained toward DA, 2D-hBN does not significantly/beneficially alter the oxidation potential of $\mathrm{MA}$ and inversely a detrimental effect on the peak current is evident (which may be due to an insulating effect of 2D-hBN toward AA). Furthermore, it has previously been shown that the oxidation mechanism of AA on carbon-based electrodes is invoked at specific functionalities and oxygenated surface sites. ${ }^{55}$ In the case reported herein, it is likely that such sites are present on the underlying electrode material and that $2 \mathrm{D}$. hBN blocks/covers these upon its immobilization, resulting in the observed decline in $\mathrm{M} \Lambda$ peak currents. For the case of $\mathrm{D} \Lambda$, the introduction of $2 \mathrm{D}-\mathrm{hBN}$ will also reduce the presence of such orygenated sites; ${ }^{25}$ however, dearly, $2 \mathrm{D}$-hBN possesses sites that support DA oxidation. This is consistent with decreasing $\mathrm{AA}$ oxidation currents with the increased mass deposition (surface coverage) of $2 \mathrm{D}-\mathrm{hBN}$, while we observe an improved electrochemical response toward DA.

We next consider the separation/resolution of DA and AA oxidation potentials at $2 \mathrm{D}-\mathrm{hBN}$ modified SPFis (in separate solutions). Figure S-14 depicts cyclic voltammograms of AA and DA utilizing unmodified and $324 \mathrm{ng} 2 \mathrm{D}-\mathrm{hBN}$ modified SPEs. Utilizing the unmodified SPE, the peak potentials for DA and AA correspond to $\sim 0.37$ and $+0.38 \mathrm{~V}$, respectively, indicating that these electrodes cannot significantly differentiate the potentials of $\mathrm{D} \Lambda$ and $\mathrm{M} .^{16,39}$ Likewise, the $2 \mathrm{D}-\mathrm{hBN}$ modified SPEs exhibit the peak potentials for $\mathrm{DA}$ and $\mathrm{AA}$ at $\sim 0.28$ and $+0.39 \mathrm{~V}$, respectively, with the beneficially reduced potential of DA and the increased potential of AA resulting in a separation of $110 \mathrm{mV}$. Given the beneficial catalytic response of $2 \mathrm{D}-\mathrm{hBN}$ modified SPEs toward $\mathrm{D} \Lambda$ and their inhibiting of $\mathrm{A}$, we next investigate if this scenario can be utilized advantageously in the simultaneous detection of DA and AA.

Differential pulse voltammetry (DPV) was utilized to explore the simultaneous detection of $\mathrm{DA}$ and $\mathrm{AA}$, with aliquots of $\mathrm{DA}$ added (representing, 3-75 $\mu \mathrm{M}$ ) into a $0.1 \mathrm{mM}$ AA solution. Figure S-15.A shows the observed voltammetry at unmodified SPFs, where upon additions of DA an increase in the current relating to the DA oxidation peak is observed (accompanying a small displacement of peak potential to more electro-negative regions). Evidently, the simultaneous detection of $\mathrm{DA}$ and $\mathrm{AA}$ is not observed. Next, we utilized a SPE with $108 \mathrm{ng}$ of 2DhBN immobilized, where (as observed in Figure S-15B) additions of DA result in a further increase in the current obtained at the DA oxidation peak. In this latter case, again, only a single peak is observed, indicating the incapability of simultaneous detection utilizing these electrodes. When comparing the linear calibration curves reported in Figure $S$. $15 \mathrm{C}$, which represent the sensitivity toward the detection of $\mathrm{DA}$, it is interesting to note that the analytical sensitivity of the 2D-hBN modified SPE is significantly increased over that of the unmodified alternative (from $4.0 \times 10^{-9} \mu \mathrm{A} \mu \mathrm{M}^{-1}$ to $2.0 \times$ $10^{-8} \mu \mathrm{A} \mu \mathrm{M}^{-1}$, respectively). Furthermore, for interested readers, Figure S-1SD illustrates that the DA oxidation potential does not significantly differ upon DA additions when utilizing a $108 \mathrm{ng} 2 \mathrm{D}$-hBN SPE, contrary to that observed at an unmodified SPE, which continually shifts more electro-negative. This suggests that $2 \mathrm{D}$ - $\mathrm{hBN}$ is less susceptible to oxidized species adsorbing onto its surface.

Although the simultaneous DPV detection of DA and $\mathrm{AA}$ is not viable, with only a single peak observed, we have dearly shown that $2 \mathrm{D}-\mathrm{hBN}$ modified SPEs (which are cheap, reproducible, portable, and analytically competitive $)^{30,41}$ could potentially be considered as an alternative/new sensor, exhibiting enhanced electroanalytical signals toward the detection of $\mathrm{D} \Lambda$.

Uric Acid and Dopamine Electrochemistry. The electrochemical detection of $1 \mathrm{mM}$ uric acid (UA) in pH 7.4 PBS was explored utilizing cyclic voltammetry at 2D-hBN modified SPEs. Inspection of Figure S-16A reveals that an unmodified SPE activates the oxidation potential of UA at $+0.49 \mathrm{~V}$, with a typical peak current of $\sim 21.1 \mu \mathrm{A}$. Upon modification of the SPE with 2D-hBN (range, 108-324 ng; see Figure S-16), it is clear that a significant alteration/change occurs in the resulting oxidation potential of UA, with an owerall reduction in the activation potential of $50 \mathrm{mV}$ toward the electro-negative region. Furthermore, the addition of $2 \mathrm{D}$ $\mathrm{hBN}$ gives rise to an increase in the observed peak current.

Next, the simultaneous detection of UA and DA was investigated via cyclic voltammetry by adding equal aliquots of $\mathrm{UA}$ and $\mathrm{DA}$ at a concentration of $0.5 \mathrm{mM}$ into a pH 7.4 PBS. Unmodified SPEs were utilized and as evident in Figure S-17A, the simultaneous detection of UA and DA is viable, with the respective oxidation peaks observed at $\sim+0.28$ and $+0.49 \mathrm{~V}$. Modification of the SPE with 2D-hBN (range, 108-324 ng; see Figure S-17A) results in a reduced peak potential for DA oxidation $(+0.19 \mathrm{~V})$, while also beneficially reducing the peak potential at which the oxidation of UA occurs $(+0.45 \mathrm{~V})$ and resultantly enhancing peak separation between $\mathrm{D} \Lambda$ and $\mathrm{U} \Lambda$ by $\sim 50 \mathrm{mV}$. Furthermore, the peak currents reconded for DA and UA were both found to increase upon the utilization of $2 \mathrm{D}$ $\mathrm{hBN}$. It is clear that $2 \mathrm{D}-\mathrm{hBN}$ modified SPFs display favorable electrocatalytic behavior toward the simultaneous detection of DA and UA, with the possibility of improved analytical sensitivity.

The optimal parameters for the combined electroanalysis of UA and DA can be identified by assessing the $\mathrm{p} K_{2}$ values of each molecule and adjusting the $\mathrm{pH}$ using the HendersonHasselbach equation. ${ }^{42}$ UA has a pK, value of $5.60 ;{ }^{45}$ therefore, at pH 7.4 the theoretical ratio of the salt to acid is 63 , meaning that UA would exist as a charge-neutral species. However, at $\mathrm{pH} 5.0$, the salt to acid ratio is 0.25 , where UA would exist in its acidic form. In the case of $\mathrm{DA}$, which exhibits a $\mathrm{pK}$ of 8.93 , ${ }^{44}$ it exists in its acidic form only when UA does not, as the mixture of the two acids will affect the acid/base equilibria between the two molecules. Therefore, at $\mathrm{pH} \mathrm{7.4,} \mathrm{DA} \mathrm{exists} \mathrm{in} \mathrm{its} \mathrm{acidic}$ form because UA is charge neutral. This is likely to affect the simultaneous detection of the analytes considerably, and we need to consider the $\mathrm{pH}$ of our experiment.

The improved voltammetry at DA and UA is likely due to favorable interaction with catalytic sites present on the 2DhBN; however, one implication that can be considered is how the charges present on the specific analytes of interest interact with and infuence the performance of the electrode material. In the case of $\mathrm{pH} 7.4$, see Figure S-17A and detailed interpretation above, we infer that the negatively charged $\mathrm{DA}^{-}$molecule is attracted to the polarized electrode, allowing for a more thermodynamically favorable charge transfer reaction, resulting in a decreased oxidation potential. Conversely, UA exhibits 

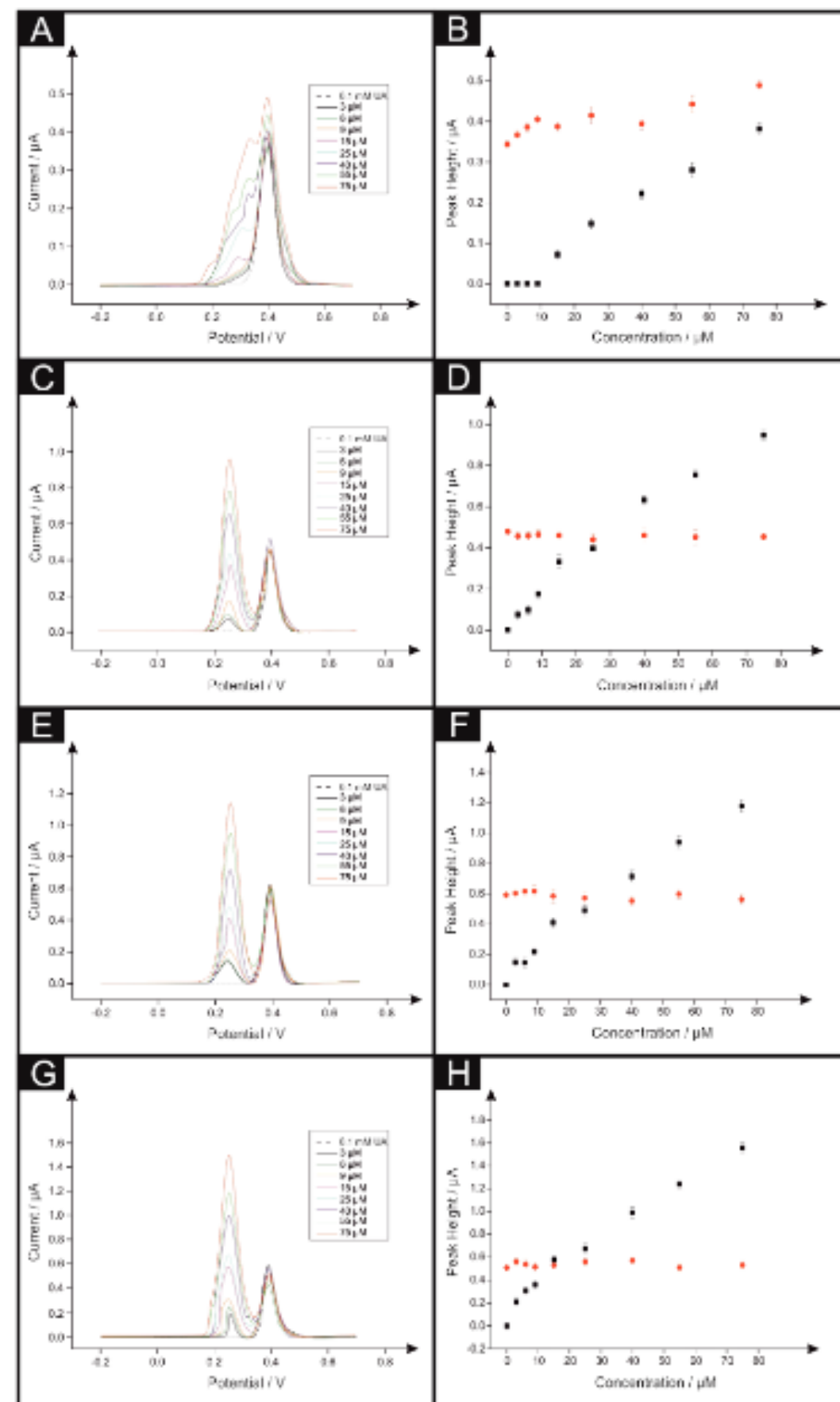

Figure 3. Typical DPVs recorded by adding aliquots of DA at concentrations in the range of 3-75 $\mu \mathrm{M}$ (in $0.1 \mathrm{mM}$ UA in pH 5.0 acetate buffer) utilizing an unmodified SPE (A) and SPEs following modification with 108 (C), 216 (E), and $324 \mathrm{ng}$ (G) $2 \mathrm{D}$-hBN. Dotted line represents 0.1 mM UA blank. Corresponding analytical curves are sbown for the simultaneous oxidation of DA (black squares) and UA (red circle) over the given concentration ranges utilizing unmodified SPFs (B) and 108 (D), 216 (F), and $324 \mathrm{ng}$ (H) 2D-hBN modified SPFs. Conditions: $F$-pulse, 20 mV; $t$ pulse, $200 \mathrm{~ms}$; equivalent scan rate, $10 \mathrm{mV} \mathrm{s}^{-1}$; (vs SCE). Fach data point (B, D, F, and II) is the average and standard deviation ( $N=3$ ).

neutral charge and will likely not be influenced to the same extent by the change in electric field at the electrodelsolution interface. For a pH 5.0 solution (sce Figure S-17B) the voltammetric scan using an unmodified SPE shows only one oxidation peak (in agreement with previous literature), ${ }^{45}$ which is likely due to the reduction of UA. However, the utilization of 
a $324 \mathrm{ng} 2 \mathrm{D}-\mathrm{hBN}$ modified SPE, results in the appearance of two oxidation peaks in pH 5.0 (at $+0.32 \mathrm{~V}$ and $+0.46 \mathrm{~V}$ for DA and UA, respectively). Evidently, the utilization of a 2D-hBN modified SPE results in the simultaneous detection of UA and $\mathrm{D} \Lambda$ at $\mathrm{pH} 5.0$, further demonstrating the electrocatalytic effect of $2 \mathrm{D}-\mathrm{hBN}$ modified SPEs toward the detection of DA in the presence of common interferents. Upon comparison of the peaks in Figure S-17A,B, at $\mathrm{pH} 5.0$ they appear sharper and exhibit improved peak resolution than the case of $\mathrm{pH} \mathrm{7.4}$, where the peaks are broader and less defined. The electroanalytical experiments henceforth were conducted at $\mathrm{pH} \mathbf{5 . 0}$.

DPV was utilized to explore the simultaneous detection of DA and UA at $\mathrm{pH} 5.0$, with aliquots of DA added (representing 3-75 $\mu \mathrm{M}$ ) into a $0.1 \mathrm{mM}$ UA (in acetate buffer) solution. Figure $3 \mathrm{~A}$ shows the observed voltammetry at unmodified SPlis, where upon additions of DA, a corresponding weak signal at $\sim+0.32 \mathrm{~V}$ is revealed (increasing in peak height respective to further additions) alongside the UA oxidation peak ( $+0.40 \mathrm{~V})$. It is notable, however, that such signal is only visible upon higher additions of DA.

Next, 2D-hBN modified SPlis (range, 108, 216, and $324 \mathrm{ng}$ ) were utilized toward the simultaneous detection of DA and UA at $\mathrm{pH}$ 5.0. Figure 3 depicts the voltammetry when utilizing $2 \mathrm{D}$ hBN modified SPEs, where two dearly separated oxidation peaks are visible at each of the modification masses utilized. Specifically, when compared to the unmodified SPE, which exhibits the oxidation potentials for DA and UA at $\sim+0.32$ and $+0.40 \mathrm{~V}$, respectively, the $2 \mathrm{D}-\mathrm{hBN}$ modified SPEs decrease (improve) the value corresponding to $\mathrm{DA}$ oxidation to $\sim 0.25$ $\mathrm{V}$ while not altering the potential at which UA oxidation is observed. In terms of the electroanalytical response, with each increased mass immobilization of $2 \mathrm{D}-\mathrm{hBN}$, it is dear through examination of Figure 3 that there is a significant enhancement of the peak current relating to the oxidation of DA, while the peak current corresponding to UA is unaffected. The values/ equations of the linear calibration curves relating to the increased analytical sensitivity toward DA detection (see Figure 3) that we observe at each of the 2D-hBN modified SPEs are presented in the Supporting Information.

In terms of the analytical utility of 2D-hBN, the $324 \mathrm{ng} 2 \mathrm{D}$. hBN modified SPE exhibited a limit of detection (IOD, based on $3 \sigma$ ) for DA (in the presence of UA) of $0.65 \mu \mathrm{M}$, which is highly competitive with the literature, as evident in Table 1. Conversely, the unmodified SPE possesses a LOD of $2.73 \mu \mathrm{M}$.

Thus, we have shown, for the first time, the utilization of $2 \mathrm{D}$. hBN modified SPEs (in pH 5.0) allows for the simultaneous detection of $\mathrm{UA}$ and $\mathrm{DA}$. Figure 3 depicts enhanced peak separations $(\sim 70 \mathrm{mV})$ and increased peak currents relating to the detection of DA (when compared to an unmodified SPE). This further suggests that $2 \mathrm{D}-\mathrm{hBN}$ modified SPFis are an effective electrocatalytic material when utilized toward the electroanalytical sensing of DA and demonstrate a continued electrocatalytic effect in the presence of UA. The observed beneficial effect is dearly dependent upon the supporting surface of $2 \mathrm{D}-\mathrm{hBN}$ and in the case of modified SPFis is likely due to the favorable interaction of $2 \mathrm{D}-\mathrm{hBN}$ with the rough surface morphology (see Figures S-8 and S-13) enabling the effective "electrical wiring" of $2 \mathrm{D}$-hBN onto the electrode surface.

Given the apparent benefits, it is interesting to consider the future potential applications of $2 \mathrm{D}-\mathrm{hBN}$ in the field of electroanalysis. A pertinent research question would be to extend the work reported herein toward "real" and more complex sample matrixes, such as in urine and cerebrospinal fluid. Moreover, fundamental studies investigating the effect of 2D-hBN lateral grain sizes on the observed electrochemistry could potentially result in defining the underlying electron transfer mechanism and the origin of this behavior, knowledge that no doubt would lead to targeted beneficial applications Currently, with the prominent deliberate doping of graphene (and other carbon-based electrodes) with (separately) boron and nitrogen atoms within the literature toward the detection of various analytes (namely, glucose, hydrogen peroxide, cocaine, and (as explored herein) $\mathrm{AA}, \mathrm{DA}$, and UA); ${ }^{14} 2 \mathrm{D}$ $\mathrm{hBN}$ offers a readily available "doped" material, which as shown in this work, can provide electrochemically useful responses. Consequently, there is clear scope to benefit multiple areas of electroanalysis through the inclusion of $2 \mathrm{D}-\mathrm{hBN}$. Furthermore, surfactants such as Nafion are widely implemented within electroanalysis to impart improvements upon fabricated sensor devices. However, it has previously been shown that such surfactants strongly influence the underlying electrochemistry, ${ }^{56}$ thus it would be beneficial for future work to consider the effects/influence of various surfactants upon the electrochemistry of $2 \mathrm{D}-\mathrm{hBN}$. With further reference to following the course of doped carbon materials, explorations of $2 \mathrm{D}-\mathrm{hBN}$ 's performance as a material component within supercapacitors may also lead to new and fascinating discoveries.

\section{CONCLUSIONS}

We have reported, for the first time, the electrocatalytic behavior of $2 \mathrm{D}-\mathrm{hBN}$ toward the detection of $\mathrm{D} \Lambda \mathrm{A}$ simple drop-casting method was implemented and the catalytic effect of $2 \mathrm{D}-\mathrm{hBN}$ was shown to be dependent upon both the mass/ coverage and substrate utilized (particularly the roughness of the underlying supporting electrode surface). Given the excellent electrocatalytic oxidation of DA observed, 2D-hBN modified SPFis were shown to possess the ability to deconvolute the signals of $\mathrm{D} \Lambda$ and two commonly reported interferents: $\mathrm{AA}$ (by $\sim 110 \mathrm{mV}$ in separate solutions) and UA (by $\sim 70$ and $50 \mathrm{mV}$ simultancously at pH 5.0 and pH 7.4 , respectively). The $2 \mathrm{D}-\mathrm{hBN}$ modified SPEs exhibited a competitive LOD value of $0.65 \mu \mathrm{M}$ and as such offer a viable means toward the detection of DA in the presence of common interferents. Evidently, this paper has shown promise for future exploration of this novel nanomaterial 2D-hBN ("an initially unilikely" candidate) as a beneficial electrode material, which may prove highly pertinent in the field of electrochemistry. 


\section{ACKNOWLEDGMENTS}

We thank the British Council for an institutional link grant (Grant No. 172726574) which supported this research. DA.C. Brownson acknowledges funding from the Ramsay Memorial Fellowships Trust.

\section{REFERENCES}

(1) Wang P.; Li, Y.; Huang X, Wang, L. Talanta 2007, 73, 431437.

(2) Jarkin, B, A. J; Fl-Sayed, M.; Brownson, D. A. Cri Banks, C. E. Anal. Methods 2012, 4, 721-729.

(3) Ali, S. R.; Ma, Y; Parajuli, R. R.; Balogun, $Y_{-;}$Lai, W. Y. C.; He, H. Anal. Chem. 2007, 79, 2583-2587.

(4) Figueiredo-Fillho, L. C. S.; Brownson, D. A. C. Fatibello-Fillo,

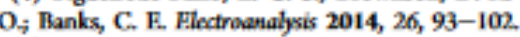

(5) Jackowska, K.; Krysinski, P. Amal. Biounal. Chem. 2013, 405, 3753-3771

(6) Brownsoe, D. A. C. Figueiredo-Fillho, L. C. S.; Rieht, B. L.j Richl, B. Di; Gomez-Mingot, M.; Iniesta, J; Fatibello-Filho, O, Banks, C. E. J. Mater. Chem. A 2016, 4, 2617-2629.

(7) Medeiros, R. A.; Benchick, A.; Rocha- Filho, R. C, Fatibello-Filho, $\mathrm{O}_{4}$ Saidani, B.; Debiemme Choury, C., Deslouis, C. Electrochem. Comman. 2012, 24, 61-64

(8) Wang, $\mathrm{Y}_{-;}$Xiao, Y. Microchim. Acta 2012, 178, 123-130.

(9) Medeiros, R. A,; Matos, R.; Benchilkh, A.; Saidani, B.; Dehiemme Chouvy, C., Deslouis, C.; Rocha Filho, R. C.; Fatibello-Filho, O. Anal Chim. Ada 2013, 797, 30-39.

(10) Deng, $\mathrm{C}_{j}$ Chen, J; Wang, M.; Xiae, $\mathrm{C}_{j}$ Nie, Z, Yao, S. Blosens Bioclectron. 2009, 24, 2091-2094.

(11) Hawley, M. D.; Tatawawadi, S. V.; Piekarski, S; Adams, R. N. J. Am. Chem. So. 1967, 89, 447-450.

(12) Filik, $H_{j}$ Avan, A. A.; Aydar, S. Arabian J. Chem. 2016, 9, 471 480,

(13) Davies, T. J; Moore, R. R.; Banks, C. E.; Compton, R. G. J. Electrounal. Chem. 2004, 574, 123-152.

(14) Bromson, D. A. C; Kampouris, D. K.; Banks, C. E. Chem. Sø. Rev. 2012, 4l, 6944-6976.

(15) Wang, II.S.; Li, T. IL.; Jia, W. La; Xn, IL.Y. Biosens Bioelectron. 2006, 22, 664-669.

(16) Wang, $Y_{j} L_{i}, Y_{j}$ Tang, $L_{i} L_{0}, J_{j}$ Li, J. Electrochem. Commum 2009, 11, 889-892.

(17) Randviir, E. P.; Brownson, D. A. C.; Banks, C. I. Mater. Today 2014, $17,426-432$

(18) Sun, H; Chao, Ji; Zoo, X.; Su, S.; Liu, X.; Yuwen, Li Fan, C Wang, L. RSC Adv. 2014, 4, 27625-27629.

(19) Zeng, IL, Zhi, C, Zhang, Z,; Wei, X,; Wang, X.; Guo, W. Bando, Y.; Golherg, D. Nano Lett 2010, 10, 5049-5055.

(20) Gao, T; Gong, L-j; Wang, Z; Yang, Z-k; Pan, W.; He, L-i Zhang, Ji Ou, E- $\mathrm{C}_{j}$ Xioeg, $\mathrm{Y}_{;}, \mathrm{X}_{\mathrm{n}}$, W. Mater. Lett. 2015, 159, 54-57.

(2.1) Xu, Q.; Cai, I,; Thao, H; Tang, J; Shen, Y.; Hu, X.; Zeng, H. Biosens. Biocelectrom. 2015, 63, 294-300.

(22) Uosaki, K.; Elumalai, G.; Noguchi, H.; Masud, T, $T_{\text {; }}$ Lyalin, $\Lambda_{\text {; }}$ Nakayama, A.; Taketsogu, T. J. Am. Chem. Sac. 2014, 136, 6542-654S,

(23) Huang, $\mathrm{C}_{\text {; }}$ Chen, C; Thang, M.; Lin, I.; Ye, X.; Lin, S.; Antonietti, M,; Wang X. Nat. Commun. 2015, 6, 7698.

(24) Gruphene Supermarket, https://graphene supermarket.com, accessed $\Lambda$ pril 1, 2015

(25) Brownson, D. A. C.; Gomez-Mingot, M.; Banks, C. E. Phys Chem. Chem. Phys 2011, 13, 20284-20288.

(26) Gorbachev, R. V.; Rizz, 1.; Nair, R. R.; Jalil, R,; Britnell, L.; Belle, B. Di; Hill, E. W.; Nowoselov, K. S, Watanabe, K.; Taniguchi, T. Geim, A. K. Blake, P. Small 2011, 7, 465-468.

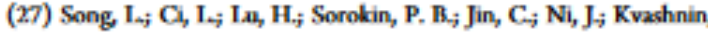
A. G.; Kvashnin, D. G.; Lou, J-; Yakobson, B. L.; Ajzyan, P. M. Nano Lett. 2010, 10, 3209-3215.

(28) Banks, C. E.; Crossley, A; Salter, C.; Wilkins, S. J.; Compton, R G. Angew. Chem, Int. Ed. 2006, 45, 2533-2537.
(29) Bhimanaputi, G. R; Koxuch, D; Robinson, J. A. Nanoscrele 2014, $6,11671-11675$

(30) Randviir, IE P.; Brownson, D. A. C; Metters, J. P.; Kadara, R. O,j Banks, C. E. Phys. Cham. Chem. Phys, 2014, 16, 4598-4611.

(31) Zhou, J; Sheng, M.; Jiang, X, Wu, G.; Gao, F. Sensons 2013, 13, 14029.

(32) Patel, $\Lambda$. N.; Tan, S-y.; Miller, T. S.; Macpherson, J. V.; Unwin, P. R. Anal Chem. 2013, 85, 11755-11764.

(33) Brownson, D. A. C, Varcy, S. A.; Hussain, F.; Ilaigh, S. JBanks, C. E. Nanoscalk 2014, 6, 1607-1621.

(34) Brownson, D. A. C. ; Kelly, P. Jij Banks, C. E. RSC Adv. 2015, S, $37281-37286$.

(35) Brownson, D. A. C.; Munro, L. J.; Kampouris, D. K,; Banks, C E. RSC Adv, 2011, 1, 978-988.

(36) Brownson, D. A. C.; Foster, C. W ; Banks, C. I. Amalys 2012, 137, 1815-1823.

(37) Rowley-Neale, S. J; Brownson, D. A. C; ; Smith, G. C.; Sawtell, D. A. G.; Kelly, P. J.; Banks, C. I. Nanoscale 2015, 7, 18152-18168

(38) Rowley-Neale, S. J.; Feam, J. M.; Brownson, D. A C.; Smith, G. $C_{i}$ Ji, X.; Banks, C. E. Nanoscale 2016, 8, 14767-14777.

(39) Cumba, L. R.; Foster, C. W.; Brownson, D. A. C; Smith, J. P.; Iniesta, J.; Thakur, B.; do Carmo, D. R.; Banks, C. E. Anabsi 2016, 141,2791 .

(40) Shin, S.; Jn, Z; Kwon, D. IL; Bose, R.; Min, Y.S. Langmuir 2015, 31, 1196-1202.

(41) Metters, J. P, Kadara, R. O ; Banks, C. E. Anulyst 2011, 136, $1067-1076$.

(42) Po, H. N.; Senoran, N. M. J. Chem. Edhe. 2001, 78, 1499.

(43) Ngo, T. C. ; Assimos, D. G. Rev. Urol. 2007, 9, 17-27.

(44) Zhong J. J. Plant Cells; Springer: Berlin, Heidelherg, Germany, 2003

(45) Kalimuthu, $\mathrm{P}_{7}$ John, S. A. Talenta 2010, 80, 1686-1691.

(46) Sheng, Z. II.; Zheng, X. Q. Xu, J.Y.; Rao, W. J.; Wang, F. B.; Xia, X.-H. Bissens. Bioeledron. 2012, 34, 125-131.

(47) Han, D; Han, T; Shan, C.j Ivaska, A, Niv, L. Electronnulysis 2010, 22, 2001-2008.

(48) Safavi, $\Lambda_{\text {; }}$ Malcki, N.; Moradlou, O.; Tajabudi, F. Anal. Biochem. 2006, 359, 224-229.

(49) Thiagarajan, S.; Chen, S.M. Talanta 2007, 74, 212-222.

(so) Lin, Li Chen, $\mathrm{J}_{i} \mathrm{Y}_{\mathrm{i}} \mathrm{a}, \mathrm{H}_{i}$ Chen, $\mathrm{Y}_{i}$ Zheng, $\mathrm{Y}_{-j}$ Lin, $\mathrm{X}$ Bioceledrochentistry 2008, 73, 11-17.

(51) Tian, X.; Cheng, C.; Yuan, IL,; Du, J; Xiao, D.; Xic, S.; Choi, M. M. F. Tulanla 2012, 93, 79-85.

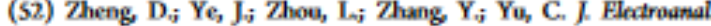
Chem. 2009, 625, 82-87.

(53) Mallesha, M; Manjunatha, R; Nethravathi, C; Suresh, G. S. Rajamathi, M.; Melo, J. S.; Venkatesha, T. V. Biadectrochemistry 2011, $81,104-108$.

(54) Khan, A F, Randviir, E. P.; Brownson, D. A C.; Ji, X.; Smith, G. C. i Banks, C. E. Electrownulysis 2016, DOI: 10.1002/elan 201600462.

(55) Hlu, I. F.; Kuwana, T. Anal Chem. 1986, 58, 3235-3239.

(56) Brownson, D. A. C.; Metters, J. P.; Kampouris, D. K.; Banks, C E. Fletrounalysis 2011, 23, 894-899. 